\title{
Resistance Profile of Klebsiella pneumoniae Strains Isolated at the Yaounde General Hospital and the Yaounde Gyneco-Obstetric and Pediatric Hospital
}

\section{Lyonga Mbamyah EE ${ }^{1,2 *}$, Teukam ACN ${ }^{1}$, Toukam $\mathbf{M}^{1}$, Djuissi M1, Enyeji $F^{1}$, MangumP ${ }^{4}$, Mesembe $\mathbf{M}^{2}$, Ikomey $\mathbf{G}^{2}$, Betbeui $\mathrm{CA}^{1,3}$ and Gonsu $\mathrm{KH}^{1,3}$}

${ }^{1}$ Department of Microbiology, Haematology, Parasitology and Infectious Diseases, Faculty of Medicine and Biomedical Sciences, University of Yaounde I, Cameroon

${ }^{2}$ Centre for the Study and Control of Communicable Diseases, Faculty of Medicine and Biomedical Sciences, University of Yaounde I, Cameroon

${ }^{3}$ Bacteriology Laboratory, University Teaching Hospital, Yaounde I, Cameroon

\section{Research Article}

Volume 6 Issue 4

Received Date: October 07, 2021

Published Date: November 12, 2021

DOI: $10.23880 /$ oajmb-16000205

${ }^{4}$ Department of Microbiology and Immunology, School of Health Sciences, Catholic University of Central Africa, Cameroon

*Corresponding author: Lyonga Mbamyah Emilia, Department of Microbiology, Haematology, Parasitology and Infectious Diseases, Faculty of Medicine and Biomedical Sciences, University of Yaounde I, Cameroon, Tel: +237678749341; Email: emilialyo@yahoo.co.uk

\section{Abstract}

Introduction: Dissemination of resistant bacteria is responsible for a considerable increase in mortality, morbidity and cost of treatment. Our study aimed to determine the frequency of Klebsiella pneumoniae infections in two referral hospitals in Yaounde Cameroon, and to examine the antibiotic resistance profile.

Methods: A cross-sectional descriptive study was carried out for a five-month period. Samples were collected from in and outpatients at the Yaounde General Hospital and at the Yaounde Gyneco-Obstetric and Pediatric Hospital. The bacteria isolation was done using standard bacteriological procedures and the identification of Klebsiella pneumoniae species was done using API 20E sytem (Biomerieux). Antibiotic susceptibility testing was determined using the disc diffusion method on Mueller Hinton media and the interpretation of the antibiogram was performed as recommended by the Comité de l'Antibiogramme de la Société Française de Microbiologie (2019). The data collected were analyzed with Epi Info 7.0 software and Excel 2013.

Results: The frequency of Klebsiella pneumoniae infections was $2.48 \%(52 / 2096)$. The majority of Klebsiella pneumoniae strains were isolated from urinary tract infections 55.77\% (29/52). Most isolates were recovered from in-patients $63.46 \%$ $(33 / 52)$ received at the pediatrics unit $25.0 \%(13 / 52)$. Few isolates were resistant to imipenem and meropenem with a resistance rate of $3.85 \%(2 / 52)$ each, while a considerable number of isolates were highly resistant to antibiotics such as ticarcillin 96.15\% (50/52), amoxicillin + clavulanic acid 94.23\% (49/52) and piperacillin $86.54 \%$ (45/52). The majority of isolates $73.08 \%$ (38/52) were multidrug-resistant and one isolate was resistant to all tested antibiotics (superbug).

Conclusion: More than half of the isolates were multidrug-resistant and one isolate from an in-patient was found to be resistant to all tested antibiotics. These findings demonstrate the importance of establishing an effective surveillance system for antimicrobial resistance in Cameroon.

Keywords: Antimicrobial Resistance Profile; Klebsiella pneumoniae 


\section{Open Access Journal of Microbiology \& Biotechnology}

Abbreviations: UTI: Urinary Tract Infection; BSI: Bloodstream Infection; PLA: Pyogenic Liver Abscess; ESBL: Extended Spectrum Beta-Lactamase; SMART: Study for Monitoring Antimicrobial Resistance Trends; EMB: Eosine Methylene Blue; CSF: Cerebrospinal Fluid; CLSI: Clinical Laboratory Standard Institute.

\section{Introduction}

Bacterial resistance to antimicrobial agents is a problem of growing importance in medical practice [1]. The spread of resistant bacteria is causing a considerable increase in mortality, morbidity and the cost of treatment [2]. Klebsiella pneumoniae is second to Escherichia coli as the most common gram-negative pathogen associated with a with diverse infections, such as urinary tract infections (UTI), bronchopneumonia, intra-abdominal infection, bloodstream infection (BSI), meningitis and pyogenic liver abscess (PLA) $[3,4]$. Among the bacteria responsible for these infections, bacteria belonging to the family Enterobacteriaceae are the most prevalent because they produce beta-lactamases and have other resistance mechanisms against antibiotics [5]. Beta-lactamases, the main enzymes responsible for resistance in Enterobacteriaceae, were described for the first time in 1983 in Germany from Klebsiella pneumoniae [6], whose resistance to antibiotics has increase dalarmingly in recent years [7]. Resistance of Gram-negative bacteria is a major public health problem [8] which is exacerbated by increasing global trends of Klebsiella species producing antibiotic resistance enzymes [9].

The global prevalence of Extended Spectrum BetaLactamase (ESBL) Klebsiella pneumoniae producers between 2005 and 2007 increased from 19.2 to $30.0 \%$ according to the Study for Monitoring Antimicrobial Resistance Trends (SMART) program [10]. Though numerous studies to assess the infectious threat posed by multidrug-resistant bacteria have been carried out so far [11,12], it is still necessary to assess the epidemiological situation in real time in order to strengthen surveillance and the control of multidrug-resistant bacteria. In this study, we report the resistance profile of Klebsiella pneumoniae strains isolated at the Yaounde General Hospital and at the Yaounde GynecoObstetric and Pediatric Hospital.

\section{Methods}

\section{Specimen}

A total of 2096 specimens were consecutively collected for bacteriological analysis within a period of five months. Specimens were collected from in and out-patients at the Yaounde General Hospital and the Yaounde Gyneco-
Obstetric and Pediatric Hospital. Klebsiella pneumoniae was isolated from specimen collected from consenting patients. The specimens analyzed included: urine, blood, pus, cerebrospinal fluid (CSF), veinous catheter and urinary catheter tips, higher-vaginal smears.

\section{Transport and Storage of Samples}

The isolates were transported from the bacteriology laboratories of the Yaounde General Hospital and the Yaounde Gyneco-Obstetric and Pediatric Hospital to the Center for Study and Control of Communicable Diseases, Faculty of Medicine and Biomedical Sciences, the University of Yaounde I, for bacteriological analysis. The isolates were transported in a cooler bag following triple packaging. These samples were sub-cultured on Eosine Methylene Blue (EMB) media to ensure their viability before being stored in brain heart infusion broth in the freezer at $-20^{\circ} \mathrm{C}$.

\section{Isolation and Identification}

Clinical specimens were cultured on Eosin Methylene Blue agarand suspected colonies were subcultured on nutrient agar to obtain pure cultures and biochemical tests were carried outusing the Api 20E identification system in accordance with the manufacturer's manual (Biomerieux SA, Lyon, France).

\section{Antimicrobial Susceptibility Testing}

This was done using the Kirby Bauer Disc diffusion method with reference to the Comite de l'Antibiogramme de la Société Française de Microbiologie (2019) performance guideline for antimicrobial susceptibility testing [13]. E. coli ATCC 25922 control strain was used to perform quality control of antibiotic disc.Twenty one antibiotics were tested, namely: amoxicillin (AMX, $25 \mu \mathrm{g}$ ), amoxicillin + clavulanic acid (AMC, 20/10 $\mu \mathrm{g}$ ), piperacillin (PIP, $30 \mu \mathrm{g}$ ), piperacillin + tazobactam (PPZ, 30/6 $\mu \mathrm{g}$ ), ticarcillin (TIC, $75 \mu \mathrm{g}$ ), ticarcillin + clavulanic acid (TCC, 75/10 $\mu \mathrm{g}$ ), cefalotin (CTT, $30 \mu \mathrm{g}$ ), cefuroxim (CFM, $30 \mu \mathrm{g}$ ), ceftazidime (CAZ, 10 $\mu \mathrm{g})$, cefotaxime (CTX, $5 \mu \mathrm{g}$ ), ceftriaxone (CRO, $30 \mu \mathrm{g}$ ), cefepime (FEP, $30 \mu \mathrm{g}$ ), imipenem (IPM, $10 \mu \mathrm{g}$ ), meropenem (MEM, $10 \mu \mathrm{g}$ ), aztreonam

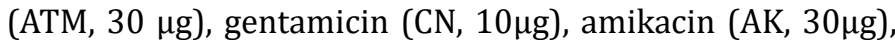
tobramycin (TM, $10 \mu \mathrm{g}$ ), norfloxacin (NOR, $10 \mu \mathrm{g}$ ); nadilixic acid (NA, $30 \mu \mathrm{g}$ ) and sulfamethoxazole + trimethoprim(STX, $1.25 / 23.75 \mu \mathrm{g})$.

\section{Detection of Multi-Drug Resistant (MDR) Isolates}

Isolates of Klebsiella pneumoniae, which showed nonsusceptibility to at least one agent in more than three 
antimicrobial classes, were considered MDR [14].

\section{Detection of Extended-Spectrum $\beta$-Lactamase (ESBL) Producing Isolates}

Screening consisted in identifying species resistant or or of intermediate susceptibility to either or both ceftazidime $(10 \mu \mathrm{g})$ and cefotaxime $(5 \mu \mathrm{g})$.Confirmation was based on the combination disc test [15].

\section{Ethical Considerations}

This study received approval from the ethics committee of the Faculty of Medicine and Biomedical Sciences of the University of Yaoundé I. All the patients signed the consent form before specimen collection. All the samples were assigned codes after collection.

\section{Results}

From the 2096 specimens collected for bacteriological analyses, 416 came from the Yaounde General Hospital and 1680 came from the Yaounde Gyneco- Obstetric and Pediatric Hospital. Among these specimens, 52 revealed a positive culture for Klebsiella pneumoniae, representing a frequency of $2.5 \%$. The majority of isolates, $63.4 \%(32 / 52)$ were from the Yaounde Gyneco-Obstetric and Pediatric Hospital and $34.6 \%(18 / 52)$ were from the Yaounde General Hospital. Klebsiella pneumoniae infections were predominant in children 0-4 years of age who had an infection rate of $32.7 \%$ $(17 / 52)$ followed by people over the age of 55 years who had an infection rate of $13.5 \%(07 / 52)$. The female population was the most infected by Klebsiella pneumoniae infections as they had an infection rate of $61.5 \%(32 / 52)$. From the 52 positive cultures for Klebsiella pneumoniae, $63.5 \%$ (33/52) isolates were from in-patients and $36.5 \%$ (19/52) from outpatients.

The frequency of Klebsiella pneumoniae in the different units in descending order was: pediatric unit $25.0 \%$ $(13 / 52)$, medicine and gynecology units, $13.5 \%(7 / 52)$ each, surgery, $9.6 \%(5 / 52)$, emergency and intensive care unit, $7.7 \%$ (4/52) each, urology, $5.8 \%(3 / 52)$, neonatal and oncology units $3.8 \%(2 / 52)$ each. The frequency of Klebsiella pneumoniae in the different clinical specimens in descending order was: urine $55.8 \%(29 / 52)$, higher vaginal smear 17.3 $\%$ (9/52), blood $11.5 \%(6 / 52)$, pus, cerebrospinal fluid (CSF), veinous catheter and urinary catheter tips $3.8 \%$ $(2 / 52)$ each and seminal fluid $1.9(1 / 52)$. These results are better illustrated in Figure 1 below.

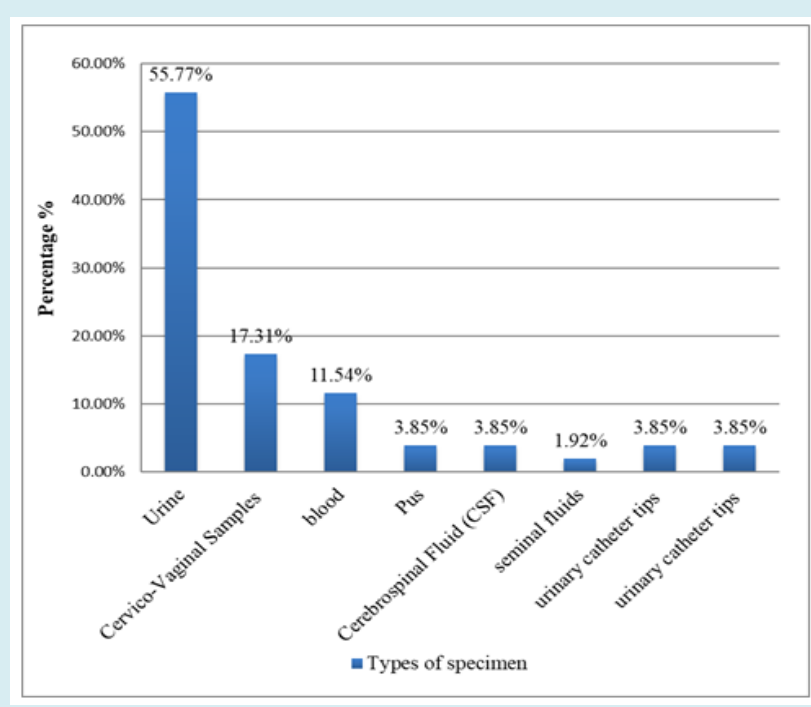

Figure 1: The prevalence of the Klebsiella pneumoniae isolates according to the different types of specimen.

Resistance to the penicillin (ticarcillin), amoxicillin + clavulanic acid, first generation cephalosporin (cephalothin), second generation cephalosporin (cefuroxime), third generation cephalosporins (ceftazidime, cefotaxime, ceftriaxone), fourth generationcephalosporin (cefepime), monobactam (aztreonam) and carbapenem (imipenem) were $96.1 \%$ (50/52), $94.2 \%(49 / 52), 78.8 \%(41 / 52)$, $75.0 \%(39 / 52), 61.5 \%(39 / 52), 46.1 \%(24 / 52), 67.3 \%$ (35/52), $57.7 \%(30 / 52), 61.5 \%(32 / 52)$ and $3.8 \%(2 / 52)$ respectively. The resistance to quinolones (nalidixic acid) and fluoroquinolones (norfloxacin) were 26.9\% (14/52) respectively. This profile is presented in Figure 2 below. 


\section{Open Access Journal of Microbiology \& Biotechnology}

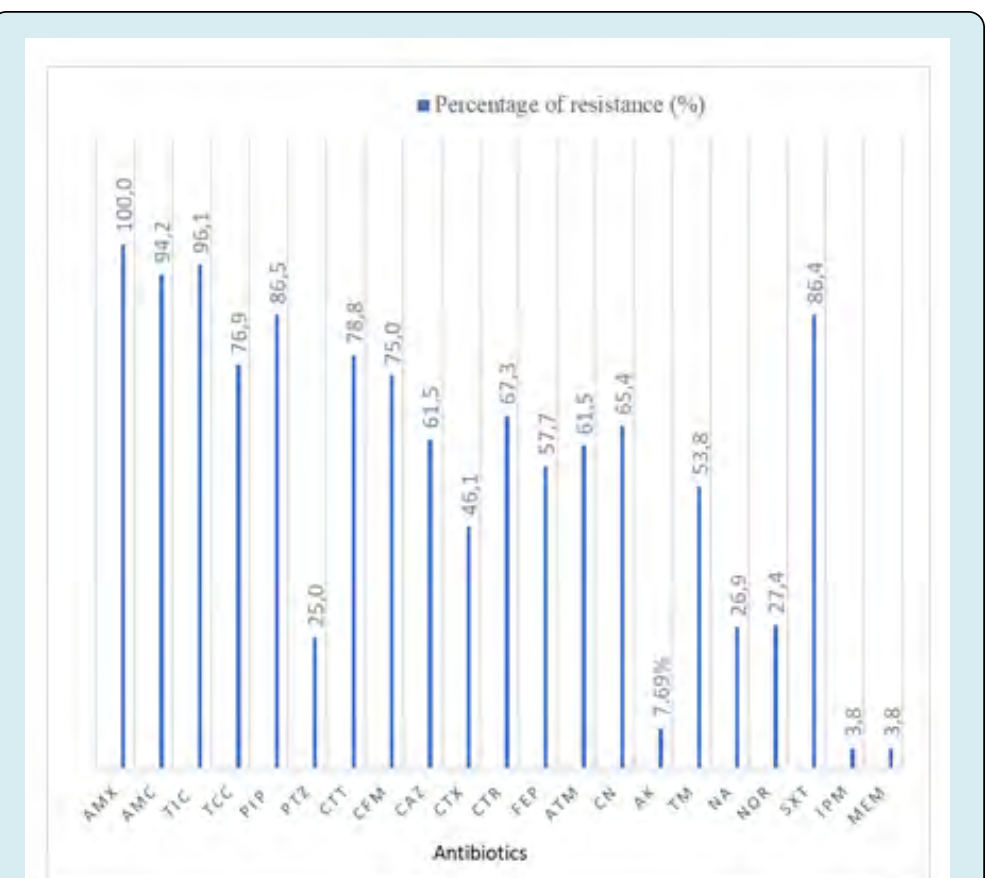

Figure 2: Resistance profile of Klebsiella pneumoniae.

\begin{tabular}{|c|c|c|}
\hline \multirow{2}{*}{ Antibiotics } & \multicolumn{2}{|c|}{ Patients } \\
\cline { 2 - 3 } & In-Patients n= 33 & Out-Patients n= 19 \\
\cline { 2 - 3 } & Percentage of resistance (\%) & Percentage of resistance (\%) \\
\hline Amoxicillin & 100,0 & 100,0 \\
\hline Amoxicillin + clavulanic acid & 97,1 & 87,50 \\
\hline Ticarcillin & 100,00 & 62,5 \\
\hline Ticarcillin + clavulanic acid & 82,3 & 75,0 \\
\hline Piperacillin & 91,2 & 6,2 \\
\hline Piperacillin + tazobactam & 32,3 & 75,0 \\
\hline Cefalotin & 85,3 & 56,2 \\
\hline Cefuroxim & 82,3 & 37,5 \\
\hline Ceftazidime & 73,5 & 18,7 \\
\hline Cefotaxime & 58,8 & 43,7 \\
\hline Ceftriaxone & 76,5 & 43,7 \\
\hline Cefepime & 64,7 & 56,2 \\
\hline Aztreonam & 61,8 & 50,0 \\
\hline Gentamicin & 70,6 & 6,2 \\
\hline Amikacin & 8,8 & 37,5 \\
\hline Tobramycine & 59,4 & 25,0 \\
\hline Nadilixic Acid & 24,2 & 13,3 \\
\hline Norfloxacin & 28,1 & 68,7 \\
\hline Imipenem & 93,9 & 0,0 \\
\hline Meropenem & 5,9 & 0,0 \\
\hline Mean & 5,9 & 45,3 \\
\hline Trimethoprim + Sulfamethoxazole & $\mathbf{6 2 , 0}$ & \\
\hline & & \\
\hline
\end{tabular}

Table 1: Resistance profile of Klebsiella pneumoniae from in-patients and out-patients. 


\section{Open Access Journal of Microbiology \& Biotechnology}

Out of the 52 isolates, the majority, 73.1\% (38/52) were multidrug-resistant and one isolate was resistant to all studied antibiotics (super bug). The frequency of multidrugresistant isolates was higher in in-patients, 68.4\% (26/38) than in out-patients $31.6 \%(12 / 38)$. However, the difference between the frequency of multidrug-resistant strains and the category of the patients was not statistically significant (p-value $=0.37)$ Table 1 .

\section{Discussion}

Our study consisted in highlighting the resistance profile of Klebsiella pneumoniae, isolated at the Yaounde General Hospital and the Yaounde Gyneco-Obstetric and Pediatric Hospital. A total of 2096 samples were collected for bacteriological analysis. We had 416 samples from the Yaounde General Hospital and 1680 samples from the Yaounde Gyneco-Obstetric and Pediatric Hospital. Out of these 2096 samples, 52 had a positive culture for Klebsiella pneumoniae, representing a prevalence of $2.5 \%$. Klebsiella pneumoniae infections were common in people over 55 years of age, $13.5 \%(07 / 52)$ and even more common in children $0-4$ years old, $32.7 \%(17 / 52)$. This can be explained in young children as a result of immaturity of their immune system and the elderly, due to weakened immune systems, hence these age groups contract infections more easily. These results are similar to those of Betbeui, et al. [12] who, worked in three reference hospitals in Cameroon on 99 isolates of Klebsiella spp, recovered $35.3 \%$ of Klebsiella pneumoniae strains in the pediatric unit. The isolates were less resistant to imipenem and meropenem, with a resistance rate of $3.8 \%(2 / 52)$ each and amikacin, $7.7 \%(4 / 52)$. The isolates were highly resistant to ticarcillin $96.1 \%(50 / 52)$, amoxicillin + clavulanic acid, $94.2 \%(49 / 52)$ and piperacillin, 86.5\% (45/52). These results are similar to those reported by Betbeui, et al. [12] who reported a Klebsiella species sensitivity rate of 99.0 $\%$ to imipenem. Our study reveals that the resistance rate was very high against amoxicilin + clavulanic acid, 94.2\% (49/52). These results corroborate those reported by Nzali, et al. [16] who found that all strains of Klebsiella pneumoniae were resistant to amoxicilin + clavulanic acid.

The majority of isolates, $73.1 \%$ (38/52) were multidrug resistant and it was reported more in isolates from inpatients, $68.4 \%(26 / 38)$ than among out- patients $31.6 \%$ $(12 / 38)$ though the difference between the frequencies and the categories of the patients, was not statistically significant ( $\mathrm{p}$-value $=0.37)$. Although, it is known that bacterial isolates from hospitalized patients harbor more resistance determinants than those isolated from community patients due to selection pressure resulting from overuse of antibiotics within hospital settings, the present study does not reveal any link. The implications of these findings are such that the treatment of infections will be more difficult and resistance mechanisms may spread to other bacteria species, causing susceptible isolates to become non-susceptible and hence therapeutic failure may occur.

\section{Conclusion}

The frequency of Klebsiella pneumoniae isolated from the different specimen was very low. Most Klebsiella pneumoniae were isolated from urinary tract infections. Most isolates were susceptible to carbapenem and highly resistant to most cephalosporins. The majority of isolates were multidrug resistant and no wild type was isolated. One isolate was resistant to all antibiotics tested. These findings demonstrate the importance of establishing an effective surveillance system for antimicrobial resistance in Cameroon.

\section{Authors' Contributions}

Emilia Enjema Lyonga conceived the study and designed it together with Aime Cesar Teukam Noukoupe and Hortense Kamga Gonsu. Emilia Enjema Lyonga, Aime Cesar Teukam Noukoupe, Modestine Djuissi, Florence Enyeji and Patience Mangum, conducted the laboratory aspect of the study with contributions from Martha Tongo Mesembe and George Mondinde Ikomey. The general supervision was carried out by Hortense Kamga Gonsu. Emilia Enjema Lyonga drafted the article with contributions from Aime Cesar Teukam Noukoupe and Martha Tongo Mesembe. All the authors reviewed the article. All the authors read and agreed to the final manuscript.

\section{Acknowledgements}

The authors wish to thank the hospital personnel at the Bacteriology Unit of the Yaounde General Hospital and the Yaounde Gyneco-Obstetric and Pediatric Hospital for the services offered during the period of specimen collection and the staff of the Centre for the Study and Control of Communicable Diseases (CSCCD), Faculty of Medicine and Biomedical Sciences, the University of Yaounde I.

\section{Funding}

This work was carried out with the aid of a grant from UNESCO and the International Development Research Center (IDRC), Ottawa, Canada. The views expressed herein do not necessarily represent those of UNESCO, IDRC or its Board of Governors.

\section{References}

1. Cohen R, Bingen E, Grimprel E, Raymond J, Gendrel D (2011) Antibiotic resistance: à new turning point not to be missed. Pan African Medical Journal 18(4): 359-361. 


\section{Open Access Journal of Microbiology \& Biotechnology}

2. Giske CG, Monnet DL, Cars O, Carmeli Y, ReAct-Action on Antibiotic Resistance (2008) Clinical and economic impact of common multidrug-resistant gram-negative bacilli. Antimicrob Agents Chemother 52(3): 813-821.

3. Podschun R, Ullmann U (1998) Klebsiella spp. as nosocomial pathogens: epidemiology, taxonomy, typing methods, and pathogenicity factors. Clin Microbiol Rev 11(4): 589-603.

4. Anderson DJ, Moehring RW, Sloane R, Schmader KE, Weber DJ, et al. (2014) Bloodstream infections in community hospitals in the 21st century: a multicenter cohort study. PLoS One 9(3): e91713.

5. Carattoli A (2009) Resistance Plasmid Families in Enterobacteriaceae. Antimicrobial Agents and Chemotherapy 53(6): 2227-2238.

6. Knothe H, Shah P, Krcmery V, Antal M, Mitsuhashi S (1983) Transferable resistance to cefotaxime, cefoxitin, cefamandole and cefuroxime in clinical isolates of Klebsiella pneumoniae and Serratia marcescens. Infection 11(6): 315-317.

7. Poirel L, Naas T, Nordmann P (2010) Diversity, epidemiology, and genetics of class $\mathrm{D}$ beta-lactamases. Antimicrob Agents Chemother 54(1): 24-38.

8. Schwaber MJ, Lidji SK, Venezia SN, Schwartz D, Leavitt A, et al. (2008) Predictors of carbapenem-resistant Klebsiella pneumoniae acquisition among hospitalized adults and effect of acquisition on mortality. Antimicrob Agents Chemother 52(3): 1028-1033.

9. Nordmann P, Cuzon G, Naas T (2009) The real threat of Klebsiella pneumoniae carbapenemase-producing bacteria. Lancet Infect Dis 9(4): 228-236.

10. Hawser SP, Bouchillon SK, Hoban DJ, Badal RE (2009) In vitro susceptibilities of aerobic and facultative anaerobic Gram-negative bacilli from patients with intra-abdominal infections worldwide from 2005-2007: results from the SMART study. Int J Antimicrob Agents 34(6): 585-588.

11. Lyonga EE, Toukam M, Nkenfou C, Gonsu HK, Assoumou MCO, et al. (2015) Resistance pattern of enterobacteriaceae isolates from urinary tract infections to selected quinolones in Yaoundé. Pan Afr Med J 21: 105.

12. Betbeui AC, Kamga HG, Toukam M, Mbakop CD, Lyonga EE, et al. (2015) Phenotypic Detection of Extended Spectrum Beta-Lactamase and Carbapenemases Produced by Klebsiella spp Isolated from Three Referrals Hospitals in Yaounde, Cameroon. Microbiology Research Journal International 9(1): 1-9.

13. CASFM / EUCAST (2019) Société Française de Microbiologie.

14. Magiorakos AP, Srinivasan A, Carey RB, Carmeli Y, Falagas ME, et al. (2012) Multidrug-resistant, extensively drug-resistant and pandrug-resistant bacteria: an international expert proposal for interim standard definitions for acquired resistance. Clin Microbiol Infect 18(3): 268-281.

15. EUCAST: Guidance documents.

16. Nzalie RNT, Gonsu HK, Shiro SK (2016) Bacterial Etiology and Antibiotic Resistance Profile of CommunityAcquired Urinary Tract Infections in a Cameroonian City. Int J Microbiol 3240268. 